\title{
An Empirical Multi-Output Production Decision Model for the Profit Maximizing Multiproduct Firm
}

\author{
Ekaterina Vorotnikova1, Serhat Asci² \\ ${ }^{1}$ Agricultural Economics and Rural Sociology Department, University of Idaho, Moscow, ID, USA \\ ${ }^{2}$ Department of Agricultural Business, California State University, Fresno, CA, USA \\ Email: evorotnikova@uidaho.edu, sasci@csufresno.edu
}

Received 30 July 2015; accepted 17 August 2015; published 20 August 2015

Copyright (C 2015 by authors and Scientific Research Publishing Inc.

This work is licensed under the Creative Commons Attribution International License (CC BY). http://creativecommons.org/licenses/by/4.0/

(c) (i) Open Access

\begin{abstract}
Empirical estimation of a theoretical multi-output production model that uses multiple inputs is difficult because of the complexities of its functional form. By using proper parameterization to linearize theoretical model's functional form, this paper develops an empirical estimation for multi-output production decision using multiple inputs in the profit maximizing firm, namely, multi-output production decision model. The model aligns with the dual approach of cost minimization and revenue maximization for the profit maximizing multi-product firm while keeping jointness in production structurally intact.
\end{abstract}

\section{Keywords}

Multiproduct Firm, Multi-Output Production Decision, Differential Production Component

\section{Introduction}

[1] developed a theory of multiproduct firm and used a differential approach to model a multi-output production using multiple inputs. They theoretically derived input demand and output supply equations for multiproduct firm. The authors also extended these derivations to the input allocation decision for the cost minimizing, the revenue maximizing firms, as well as multi-output production decision for the profit maximizing firms. However, the complexities of the equations presented some barriers to empirical estimation of such models. This paper developed an empirical estimation model for multi-output production decision using multiple inputs in the profit maximizing firm, namely, multi-output production decision model.

[2] comprehensively derived input demand and output supply systems and exemplified the estimation of these 
systems. However, he recommended imposing input-output separability restriction to simplify the input allocation and multi-output production decision models for estimation. This restriction resulted in input allocation decision independent of the changes in output prices and multi-output production decision independent of the changes in the input prices, which was counterproductive to the multiproduct firm theory.

Recently, [3] and [4] developed empirical models to estimate input allocation in the revenue maximizing and the cost minimizing firms, respectively. These empirical models linearized functional forms of the input allocation models without having to impose the input-output separability and input independence restrictions. Additionally, the authors suggested a statistical test, which checked whether imposing these restrictions was necessary. By using this linearization technique, we developed an empirical model for the multi-output production decision for the profit maximizing firm that combined both cost minimization and revenue maximization for a particular firm. We also proved homogeneity property for the individual input price parameter for each output.

[5] and [6] estimated output-supply model. However, no one to date had empirically estimated multi-output production decision model due to the complexities associated with the input price terms. This paper reformulated multi-output production decision model for the profit maximizing firm by using proper parameterization to linearize theoretical model's functional form. This advancement allowed us develop multi-output production decision model that could easily be estimated empirically.

The empirical model suggested that multi-output production decision was a function of the Divisia output volume index and the relative changes in the individual input and output prices. The model was derived in such a way that the theoretical adding-up conditions held for all parameters. The restrictions like homogeneity, symmetry, input-output separability and output independence cpuld be imposed and tested statistically.

The paper was organized in the following way: Section 2 developed the multi-output production decision model for profit maximizing firm. Section 3 linearized and reformulated the model empirically, and Section 4 concluded the paper.

\section{Multi-Output Production Decision Model for Profit Maximizing Firm}

Profit maximizing multiproduct firm implies the following multi-output production decision equation for the $r$ th product [1] [2]

$$
g_{r} d\left(\ln z_{r}\right)=\theta_{r}^{*} d(\ln Z)-\psi^{*} \sum_{s} \theta_{r s}^{*} d\left(\ln \frac{p_{s} / W^{\prime s}}{P^{\prime} / W^{\prime \prime}}\right)
$$

where $g_{r}=p_{r} z_{r} / \sum_{r} p_{r} z_{r}$ is the $r$ th output's share in revenue; $z_{r}$ is the quantity of the $r$ th output; $p_{r}$ is the price of the $r$ th output $(r, s=1,2, \cdots, m) ; d(\ln Z)=\sum_{r} g_{r} d\left(\ln z_{r}\right)$ is a Divisia output volume index;

$d\left(\ln P^{\prime}\right)=\sum_{r} \theta_{r}^{*} d\left(\ln p_{r}\right)$ is a Frisch output price index where $p_{r}$ is the price of the $r$ th output;

$d\left(\ln W^{\prime s}\right)=\sum_{i} \theta_{i}^{s} d\left(\ln w_{i}\right)$ and $d\left(\ln W^{\prime \prime}\right)=\sum_{s} \theta_{s}^{*} d\left(\ln W^{\prime s}\right)$ are Frisch input price indexes where $w_{i}$ is the price of the ith input $(i=1,2, \cdots, n)$. The coefficient $\theta_{r s}^{*}$ is normalized output price coefficient and represents a pure substitution effect between the $r$ th and sth products. Therefore, we define $\Theta^{*}=\left[\theta_{r s}^{*}\right]$ is an $m \times m$ symmetric positive definite matrix, $\sum_{r} \sum_{s} \theta_{r s}^{*}=\sum_{r} \theta_{r}^{*}=1$. In addition, $\theta_{r}^{*}=\sum_{i} \bar{\theta}_{i} \bar{\theta}_{r}^{i}, \bar{\theta}_{r}^{i}=\partial\left(p_{r} z_{r}\right) / \partial\left(w_{i} q_{i}\right)$ is described as the $r$ th product marginal revenue for the ith input. $\theta_{i}^{s}=\partial\left(w_{i} q_{i}\right) / \partial\left(p_{s} z_{s}\right)$ expresses the additional cost of the ith input used in the production relative to the additional dollar's value of the sth output as a necessary condition for profit maximization. $\bar{\theta}_{i}=\sum_{r} \theta_{r}^{*} \theta_{i}^{r}$ is such that $\sum_{i} \sum_{j} \bar{\theta}_{i j}=\sum_{i} \bar{\theta}_{i}=1$, where $\bar{\theta}_{i j}$ are normalized coefficients and $\sum_{i} \theta_{i}^{r}=1[2]$.

Finally, the revenue-cost ratio is $\gamma=R / C$ where $R=\sum_{r} p_{r} z_{r}$ is the firm's revenue, and $C=\sum_{i} w_{i} q_{i}$ is the cost. The $\psi^{*}$ is the price elasticity of the supply and defined by $(1 / R) \mathbf{p}^{\prime}\left(\partial^{2} C /\left(\partial \mathbf{z} \partial \mathbf{z}^{\prime}\right)\right)^{-1} \mathbf{p}>0$. It also satisfies $\psi^{*} \geq \psi /(\gamma-\psi)>0$, where $\psi$ is a measure of the curvature of the logarithmic cost function and can be found using $1+\left(1 / \gamma^{2}\right) \sum_{r} \sum_{s}\left(\partial^{2} \ln C /\left(\partial \ln z_{r} \partial \ln z_{s}\right)\right)$ for output homogeneous production function [2]. By these definitions, Equation (1) shows that the multi-output production decision is affected by the changes in both output and input prices. 
Note that when the firm is input-output separable, the multi-output production decision model becomes independent of the input price changes. This restriction implies that the individual input price indexes are the same for each output. Hence, the Frisch input price indexes are equal to each other, $d\left(\ln W^{\prime s}\right)=d\left(\ln W^{\prime \prime}\right)=d\left(\ln W^{\prime}\right)$. Therefore, the input prices disappear in the Equation (1). Further output independence restriction yields $\theta_{r s}^{*}=0$ for $r \neq s$ and $\theta_{r s}^{*}=\theta_{r}^{*}>0$ for $r=s[2]$.

\section{Multi-Output Production Decision Model}

\subsection{Linear Model}

This section simplifies Equation (1) to a linear form. We can show Equation (1) as the three-term summation:

$$
g_{r} d\left(\ln z_{r}\right)=\theta_{r}^{*} d(\ln Z)-\psi^{*} \sum_{s} \theta_{r s}^{*} d\left(\ln \frac{p_{s}}{P^{\prime}}\right)-\psi^{*} \sum_{s} \theta_{r s}^{*} d\left(\ln \frac{W^{\prime \prime}}{W^{\prime s}}\right)
$$

When we decompose output price terms into two terms using the above definition, $-\psi^{*} \sum_{s} \theta_{r s}^{*} d\left(\ln p_{s} / P^{\prime}\right)$ can be written as

$$
-\psi^{*} \sum_{s} \theta_{r s}^{*} d\left(\ln p_{s}\right)+\psi^{*} \theta_{r}^{*} \sum_{s} \theta_{s}^{*} d\left(\ln p_{s}\right)
$$

Collecting under output price term, the two terms can be written as

$$
-\psi^{*} \sum_{s}\left(\theta_{r s}^{*}-\theta_{r}^{*} \theta_{s}^{*}\right) d\left(\ln p_{s}\right)=\sum_{s} \pi_{r s}^{*} d\left(\ln p_{s}\right)
$$

where $\pi_{r s}^{*}$ can obey the adding-up condition, $\sum_{r} \pi_{r s}^{*}=0$, the homogeneity condition, $\sum_{s} \pi_{r s}^{*}=0$, and the symmetry restriction, $\pi_{r s}^{*}=\pi_{s r}^{*} \forall r, s$.

Then, we rewrite the last term of Equation (2) as, $-\psi^{*} \sum_{s} \theta_{r s}^{*}\left(d\left(\ln W^{\prime \prime}\right)-d\left(\ln W^{\prime s}\right)\right)$ and substitute $d\left(\ln W^{\prime \prime}\right)=\sum_{s} \theta_{s}^{*} d\left(\ln W^{\prime s}\right)$ in this expression

$$
-\psi^{*} \sum_{s} \theta_{r s}^{*} \sum_{s} \theta_{s}^{*} d\left(\ln W^{\prime s}\right)+\psi^{*} \sum_{s} \theta_{r s}^{*} d\left(\ln W^{\prime s}\right)
$$

By using $\sum_{s} \theta_{r s}^{*}=\theta_{r}^{*}$ and the former term of expression (5) simplifies to $-\psi^{*} \sum_{s} \theta_{r}^{*} \theta_{s}^{*} d\left(\ln W^{\prime s}\right)$, as shown in Appendix A. Next, we insert this term into expression (5), and by rearranging the terms, it yields

$$
\psi^{*} \sum_{s}\left(\theta_{r s}^{*}-\theta_{r}^{*} \theta_{s}^{*}\right) d\left(\ln W^{\prime s}\right)
$$

We substitute $d\left(\ln W^{\prime s}\right)=\sum_{i} \theta_{i}^{s} d\left(\ln w_{i}\right)$ into the expression (6) and obtain

$$
\psi^{*} \sum_{s}\left(\theta_{r s}^{*}-\theta_{r}^{*} \theta_{s}^{*}\right) \sum_{i} \theta_{i}^{s} d\left(\ln w_{i}\right)
$$

By distributive property, we rewrite expression (7) as

$$
\psi^{*} \sum_{s} \theta_{r s}^{*} \sum_{i} \theta_{i}^{s} d\left(\ln w_{i}\right)-\psi^{*} \sum_{s} \theta_{r}^{*} \theta_{s}^{*} \sum_{i} \theta_{i}^{s} d\left(\ln w_{i}\right)
$$

$\psi^{*} \sum_{s} \theta_{r s}^{*} \sum_{i} \theta_{i}^{s} d\left(\ln w_{i}\right)$ can be simplified to $\psi^{*} \sum_{i}\left(\sum_{s} \theta_{r s}^{*} \theta_{i}^{s}\right) d\left(\ln w_{i}\right)$, as shown in Appendix A. Since $\sum_{s} \theta_{s}^{*} \theta_{i}^{s}=\bar{\theta}_{i}$, we define $\sum_{s} \theta_{r s}^{*} \theta_{i}^{s}=\bar{\theta}_{r i}$. We can then rewrite the first term of expression (8) as $\psi^{*} \sum_{i} \bar{\theta}_{r i} d\left(\ln w_{i}\right)$, where $\theta_{i r}^{*}$ represents the revenue gained by the firm from the additional production of the $r$ th product for ith input [3]. We can simplify $-\psi^{*} \sum_{s} \theta_{r}^{*} \theta_{s}^{*} \sum_{i} \theta_{i}^{s} d\left(\ln w_{i}\right)$ by taking $\theta_{r}^{*}$ outside of the sum and $\theta_{s}^{*}$ inside of the sum; thus, we end up with $-\psi^{*} \theta_{r}^{*} \sum_{i} \sum_{s} \theta_{s}^{*} \theta_{i}^{s} d\left(\ln w_{i}\right)$. Using the definition $\sum_{s} \theta_{s}^{*} \theta_{i}^{s}=\bar{\theta}_{i}$, we obtain $-\psi^{*} \theta_{r}^{*} \sum_{i} \bar{\theta}_{i} d\left(\ln w_{i}\right)=-\psi^{*} \sum_{i} \theta_{r}^{*} \bar{\theta}_{i} d\left(\ln w_{i}\right)$.

Substituting simplified terms into expression (8) yields

$$
\psi^{*} \sum_{i} \bar{\theta}_{r i} d\left(\ln w_{i}\right)-\psi^{*} \sum_{i} \theta_{r}^{*} \bar{\theta}_{i} d\left(\ln w_{i}\right)=\psi^{*} \sum_{i}\left(\bar{\theta}_{r i}-\theta_{r}^{*} \bar{\theta}_{i}\right) d\left(\ln w_{i}\right)
$$


Define $\bar{\pi}_{r i}=\bar{\psi}\left(\bar{\theta}_{r i}-\theta_{r}^{*} \bar{\theta}_{i}\right)$ and substitute expressions (4) and (9) into Equation (2). Thus, the linear form of the multi-output production decision equation becomes

$$
g_{r} d\left(\ln z_{r}\right)=\theta_{r}^{*} d(\ln Z)+\sum_{s} \pi_{r s}^{*} d\left(\ln p_{s}\right)+\sum_{i} \bar{\pi}_{r i} d\left(\ln w_{i}\right)
$$

The properties of the parameters are demonstrated in Appendix B. In summary, all parameters automatically hold the adding-up condition, $\sum_{r} \theta_{r}^{*}=1, \sum_{r} \pi_{r s}^{*}=0$, and $\sum_{r} \sum_{i} \bar{\pi}_{r i}=0$. Next, one can impose homogeneity condition on price parameters, $\sum_{s} \pi_{r s}^{*}=0$ and $\sum_{i} \bar{\pi}_{r i}=0$. The symmetry restriction only holds for output price parameters $\pi_{r s}=\pi_{s r} \forall r, s$. Homogeneity condition is proved as a property of parameter $\bar{\pi}_{r i}$ although previous study by [4] could not confirm homogeneity condition for this parameter. It is worth noting that symmetry in the $n \times m$ matrix $\bar{\pi}=\left[\bar{\pi}_{r i}\right]$ is not necessary to hold.

\subsection{An Empirical Model}

We parameterize Equation (10) by assuming $\theta_{r}^{*}, \pi_{r s}^{*}$ and $\bar{\pi}_{r i}$ are constants and add disturbance $\varepsilon_{r}^{*}$ for empirical estimation, where $\varepsilon_{r}^{*}$ is $m$-variate normal distribution with zero means [2]. Thus, the empirical model for multi-output production decision in a profit maximizing firm that uses multiple inputs is

$$
\bar{g}_{r t} d z_{r}=\theta_{r}^{*} d Z_{t}+\sum_{s} \pi_{r s}^{*} d p_{s t}+\sum_{i} \bar{\pi}_{r i} d w_{i t}+\varepsilon_{r t}^{*}
$$

To estimate parameter, we calculate arithmetic means of output shares, $\bar{g}_{r t}=\left(g_{r, t}+g_{r, t-1}\right) / 2$; log difference of quantity and prices, $d x_{t}=\ln x_{t}-\ln x_{t-1}$ with $x$ representing $z, p$ and $w$; Divisia volume index, $d Z_{t}=\sum_{r} \bar{g}_{r t} d z_{r t}$; and include $\varepsilon^{*} \sim N(0, \Sigma)$.

The model naturally maintains the adding-up conditions. Symmetry conditions on $\pi^{*}$ and homogeneity conditions on $\pi^{*}=\left[\pi_{r s}^{*}\right]$ and $\bar{\pi}$ are imposable. Log-likelihood-ratio tests (LRT) can test these restrictions. The covariance matrix, $\Sigma$, happens to be singular due to the adding-up conditions.

After dropping one equation we estimate the remaining $m-1$ equations simultaneously [7]. Maximum likelihood or iterative seemingly unrelated regression (SUR), which also iterates to maximum likelihood [8] [9], can estimate the parameters $\boldsymbol{\theta}^{*}=\left[\theta_{r}^{*}\right], \pi^{*}$ and $\overline{\boldsymbol{\pi}}$. By imposing input-output separability restriction we get

$$
\bar{g}_{r t} d z_{r}=\theta_{r}^{*} d Z_{t}+\sum_{s} \pi_{r s}^{*} d p_{s t}+\varepsilon_{r t}^{*}
$$

where input prices disappear from the linear form. Further output separability restriction simplifies Equation (12) to

$$
\bar{g}_{r t} d z_{r}=\theta_{r}^{*} d Z_{t}-\psi^{*} \theta_{r}^{*} d\left(\ln \frac{p_{s t}}{P_{t}^{\prime}}\right)+\varepsilon_{r t}^{*}
$$

Both restrictions can be tested with an LRT.

\section{Conclusions}

This study makes it possible to empirically estimate the decision model that optimizes the production process with multiple inputs being used across multiple outputs. The model aligns with the dual approach of cost minimization and revenue maximization for the profit maximizing multi-product firm while keeping jointness in production structurally intact.

We reformulate multi-output production decision model by using proper parameterization to linearize theoretical model's functional form. Homogeneity property for the individual input price parameter for each output is formally proven, which is never done before.

\section{References}

[1] Laitinen, K. and Theil, H. (1978) Supply and Demand of the Multiproduct Firm. European Economic Review, 11, 107154. http://dx.doi.org/10.1016/0014-2921(78)90031-4 
[2] Laitinen, K. (1980) A Theory of the Multiproduct Firm. In: Theil, H. and Glejser, H., Eds., Studies in Mathematical and Managerial Economics, North Holland, New York, 28.

[3] Seale Jr., J.L., Vorotnikova, E. and Asci, S. (2014) An Empirical Input Allocation Model for the Multiproduct Firm. Economics Letters, 124, 367-369. http://dx.doi.org/10.1016/j.econlet.2014.06.021

[4] Zhu, M., Onel, G. and Seale Jr., J.L. (2015) An Empirical Input Allocation Model for the Cost Minimizing Multiproduct Firm. Economics Letters, 132, 73-76. http://dx.doi.org/10.1016/j.econlet.2015.04.015

[5] Clements, K.W. (1980) An Aggregative Multiproduct Supply Model. European Economics Review, 13, $239-245$. http://dx.doi.org/10.1016/0014-2921(80)90057-4

[6] Rossi, N. (1984) The Estimation of the Product Supply and Input Demand by the Differential Approach. American Journal of Agricultural Economics, 66, 368-375. http://dx.doi.org/10.2307/1240804

[7] Barten, A.P. (1977) The Systems of Consumer Demand Functions Approach: A Review. Econometrica, 45, 23-50. http://dx.doi.org/10.2307/1913286

[8] Harvey, A. (1990) The Econometric Analysis of Time Series. 2nd Edition, The MIT Press, Cambridge.

[9] Kmenta, J. and Gilbert, R.F. (1968) Small Sample Properties of Alternative Estimators of Seemingly Unrelated Regressions. Journal of the American Statistical Association, 63, 1180-1200. http://dx.doi.org/10.1080/01621459.1968.10480919 


\section{Appendix A: Simplification of Summation Terms}

The expression $-\psi^{*} \sum_{s} \theta_{r s}^{*} \sum_{s} \theta_{s}^{*} d\left(\ln W^{\prime s}\right)$ can be written as

$$
\begin{aligned}
& -\psi^{*}\left[\theta_{1}^{*}\left(\theta_{r 1}^{*}+\cdots+\theta_{r m}^{*}\right) d\left(\ln W^{\prime 1}\right)+\cdots+\theta_{m}^{*}\left(\theta_{r 1}^{*}+\cdots+\theta_{r m}^{*}\right) d\left(\ln W^{\prime m}\right)\right] \\
& =-\psi^{*}\left[\theta_{1}^{*} \sum_{s} \theta_{r s}^{*} d\left(\ln W^{\prime 1}\right)+\cdots+\theta_{m}^{*} \sum_{s} \theta_{r s}^{*} d\left(\ln W^{\prime m}\right)\right] .
\end{aligned}
$$

Using $\sum_{s} \theta_{r s}^{*}=\theta_{r}^{*}$, it simplifies to $-\psi^{*} \sum_{s} \theta_{r}^{*} \theta_{s}^{*} d\left(\ln W^{\prime s}\right)$.

In explicit form, $\psi^{*} \sum_{s} \theta_{r s}^{*} \sum_{i} \bar{\theta}_{i}^{s} d\left(\ln w_{i}\right)$ can be written as

$$
\psi^{*}\left[\theta_{r 1}^{*}\left(\bar{\theta}_{1}^{1} d\left(\ln w_{1}\right)+\cdots+\bar{\theta}_{n}^{1} d\left(\ln w_{n}\right)\right)+\cdots+\theta_{r m}^{*}\left(\bar{\theta}_{1}^{m} d\left(\ln w_{1}\right)+\cdots+\bar{\theta}_{n}^{m} d\left(\ln w_{n}\right)\right)\right] .
$$

Further rearrangement and simplification, it yields

$$
\psi^{*}\left[\left(\theta_{r 1}^{*} \bar{\theta}_{1}^{1}+\theta_{r 2}^{*} \bar{\theta}_{1}^{2}+\cdots+\theta_{r m}^{*} \bar{\theta}_{1}^{m}\right) d\left(\ln w_{1}\right)+\cdots+\left(\theta_{r 1}^{*} \bar{\theta}_{n}^{1}+\theta_{r 2}^{*} \bar{\theta}_{n}^{2}+\cdots+\theta_{r m}^{*} \bar{\theta}_{n}^{m}\right) d\left(\ln w_{n}\right)\right],
$$

and we can write this simplified expression as $=\psi^{*} \sum_{i}\left(\sum_{s} \theta_{r s}^{*} \bar{\theta}_{i}^{s}\right) d\left(\ln w_{i}\right)$.

\section{Appendix B: Properties of Parameters}

Define $\pi_{r s}^{*}=-\psi^{*}\left(\theta_{r s}^{*}-\theta_{r}^{*} \theta_{s}^{*}\right)$. Sum $\pi_{r s}^{*}$ over $r$, which leads to

$$
\sum_{r} \pi_{r s}^{*}=-\psi^{*} \sum_{r}\left(\theta_{r s}^{*}-\theta_{r}^{*} \theta_{s}^{*}\right)=-\psi^{*} \sum_{r} \theta_{r s}^{*}+\psi^{*} \theta_{s}^{*} \sum_{r} \theta_{r}^{*}=-\psi^{*} \theta_{s}^{*}+\psi^{*} \theta_{s}^{*}=0
$$

and hence, $\pi_{r s}^{*}$ obeys the adding-up condition. To show the homogeneity condition, sum $\pi_{r s}^{*}$ over $s$

$$
\sum_{s} \pi_{r s}^{*}=-\psi^{*} \sum_{s}\left(\theta_{r s}^{*}-\theta_{r}^{*} \theta_{s}^{*}\right)=-\psi^{*} \sum_{s} \theta_{r s}^{*}+\psi^{*} \theta_{r}^{*} \sum_{s} \theta_{s}^{*}=-\psi^{*} \theta_{r}^{*}+\psi^{*} \theta_{r}^{*}=0
$$

Lastly, symmetry holds for $m \times m$ matrix, where $\pi_{r s}^{*}=\pi_{s r}^{*} \forall r, s$.

Define $\bar{\theta}_{r i}=\sum_{s} \theta_{r s}^{*} \theta_{i}^{s}$. Sum $\bar{\theta}_{r i}$ over both $i$ and $r$ to show it as a normalized input price parameter. Using [2] definition $\sum_{s} \theta_{s}^{*} \theta_{i}^{s}=\bar{\theta}_{i}$, we find

$$
\sum_{i} \sum_{r} \bar{\theta}_{r i}=\sum_{i} \sum_{r}\left(\sum_{s} \theta_{r s}^{*} \theta_{i}^{s}\right)=\sum_{i} \sum_{s}\left(\sum_{r} \theta_{r s}^{*}\right) \theta_{i}^{s}=\sum_{i} \sum_{s} \theta_{s}^{*} \theta_{i}^{s}=\sum_{i} \bar{\theta}_{i}=1 .
$$

Individually summing over $r$ and $i$, respectively, yields

$$
\sum_{r} \bar{\theta}_{r i}=\sum_{r}\left(\sum_{s} \theta_{r s}^{*} \theta_{i}^{s}\right)=\sum_{s}\left(\sum_{r} \theta_{r s}^{*}\right) \theta_{i}^{s}=\sum_{s} \theta_{s}^{*} \theta_{i}^{s}=\bar{\theta}_{i}
$$

and

$$
\sum_{i} \bar{\theta}_{r i}=\sum_{i} \sum_{s}\left(\theta_{r s}^{*} \theta_{i}^{s}\right)=\sum_{s}\left(\sum_{i} \theta_{i}^{s}\right) \theta_{r s}^{*}=\sum_{s} \theta_{r s}^{*}=\theta_{r}^{*}
$$

for the parameter with $m \times n$ dimension.

Define $\bar{\pi}_{r i}=\bar{\psi}\left(\bar{\theta}_{r i}-\theta_{r}^{*} \bar{\theta}_{i}\right)$. Sum $\pi_{r s}^{*}$ over $r$ which leads to

$$
\sum_{r} \bar{\pi}_{r i}=\bar{\psi} \sum_{r}\left(\bar{\theta}_{r i}-\theta_{r}^{*} \bar{\theta}_{i}\right)=\bar{\psi} \sum_{r} \bar{\theta}_{r i}-\bar{\psi} \bar{\theta}_{i} \sum_{r} \theta_{r}^{*}=\bar{\psi} \bar{\theta}_{i}-\bar{\psi} \bar{\theta}_{i}=0
$$

and hence, $\bar{\pi}_{r i}$ obeys the adding-up condition. To show the homogeneity condition, sum $\bar{\pi}_{r i}$ over $i$

$$
\begin{aligned}
\sum_{i} \bar{\pi}_{r i} & =\bar{\psi} \sum_{i}\left(\bar{\theta}_{r i}-\theta_{r}^{*} \bar{\theta}_{i}\right)=\bar{\psi} \sum_{i} \bar{\theta}_{r i}-\bar{\psi} \sum_{i} \theta_{r}^{*} \bar{\theta}_{i}=\bar{\psi} \theta_{r}^{*}-\bar{\psi} \sum_{i}\left(\sum_{i} \bar{\theta}_{i} \bar{\theta}_{r}^{i}\right) \bar{\theta}_{i} \\
& =\bar{\psi} \theta_{r}^{*}-\bar{\psi} \sum_{i} \bar{\theta}_{i} \bar{\theta}_{r}^{i}\left(\sum_{i} \overline{\theta_{i}}\right)=\bar{\psi} \theta_{r}^{*}-\bar{\psi} \sum_{i} \bar{\theta}_{i} \bar{\theta}_{r}^{i}=\bar{\psi} \theta_{r}^{*}-\bar{\psi} \theta_{r}^{*}=0 .
\end{aligned}
$$

Lastly, symmetry does not hold for $m \times n$ matrix. 